\title{
Response Function of the BGO and NaI(T1) Detectors Using Monte Carlo Simulations
}

\author{
I. ORION ${ }^{a, b, c}$ AND L. WIELOPOLSKI ${ }^{b}$ \\ ${ }^{a}$ St. Luke's-Roosevelt Hospital, Columbia University, New York, \\ New York 10025, USA \\ ${ }^{b}$ Brookhaven National Laboratory, Department of Applied Science, \\ Upton, New York 11973, USA
}

\section{INTRODUCTION}

The high efficiency of the BGO detectors makes them very attractive candidates to replace $\mathrm{NaI}(\mathrm{Tl})$ detectors, which are widely used in studies of body composition. In this work, the response functions of the $\mathrm{BGO}$ and $\mathrm{NaI}(\mathrm{Tl})$ detectors were determined at $0.662,4.4$, and $10.0 \mathrm{MeV}$ using three different Monte Carlo codes: EGS4, ${ }^{1}$ $\mathrm{MCNP}^{2}$ and PHOTON. ${ }^{3}$ These codes differ in their input files and transport calculations, and were used to verify the internal consistency of the setup and of the input data. The energy range of 0.662 to $10 \mathrm{MeV}$ was chosen to cover energies of interest in body composition studies. The superior efficiency of the BGO-detectors has to be weighed against their inferior resolution, and their higher price than that of the $\mathrm{NaI}$ detectors. Because the price of the BGO detectors strongly depends on the size of the crystal, its optimization is an important component in the design of the entire system.

\section{METHOD}

The intrinsic response functions were calculated for a point source at $10 \mathrm{~cm}$ from the base of a cylindrical detector, where the source emission was forced into the solid angle subtended by the detector. The energy deposition in the detector-that is, the photon spectrum-was calculated using 512 bins $20 \mathrm{keV}$ wide. Special attention was paid to the energy cutoff used in the codes. Although this value is fixed at $10 \mathrm{keV}$ in the PHOTON code, to improve accuracy we altered it in the other two codes to $1 \mathrm{keV}$. The main effect of the cut-off value is in the valley area beside the photopeak (FIG. 1a). The calculated photon spectra were convoluted with an energy resolution function of the type $a+b E^{1 / 2}+c E$ that was fitted to the experimental data for cach detector reported in the literature. ${ }^{4}$ The convolution consisted of Monte Carlo normal sampling of each energy bin. We found that from about $2 \mathrm{MeV}$ and above, the results from the PHOTON code deviated systematically from the results obtained using the other two codes (FIG. 1a, b, and c). We attributed this discrepancy to inadequate bremsstrahlung cross-sections used in the PHOTON code (we are verifying this with the authors of the code). The PHOTON code was not used further at high energies.

\footnotetext{
${ }^{c}$ Address for correspondence: Itzhak Orion, Ph.D., Brookhaven National Laboratory, Medical Department, Bell Avenue, Upton, NY 11973. Voice: 516-344-3653; fax: 516-344-5311. orion@bnl.gov, This work was sponsored under the auspices of U.S.DOE.
} 

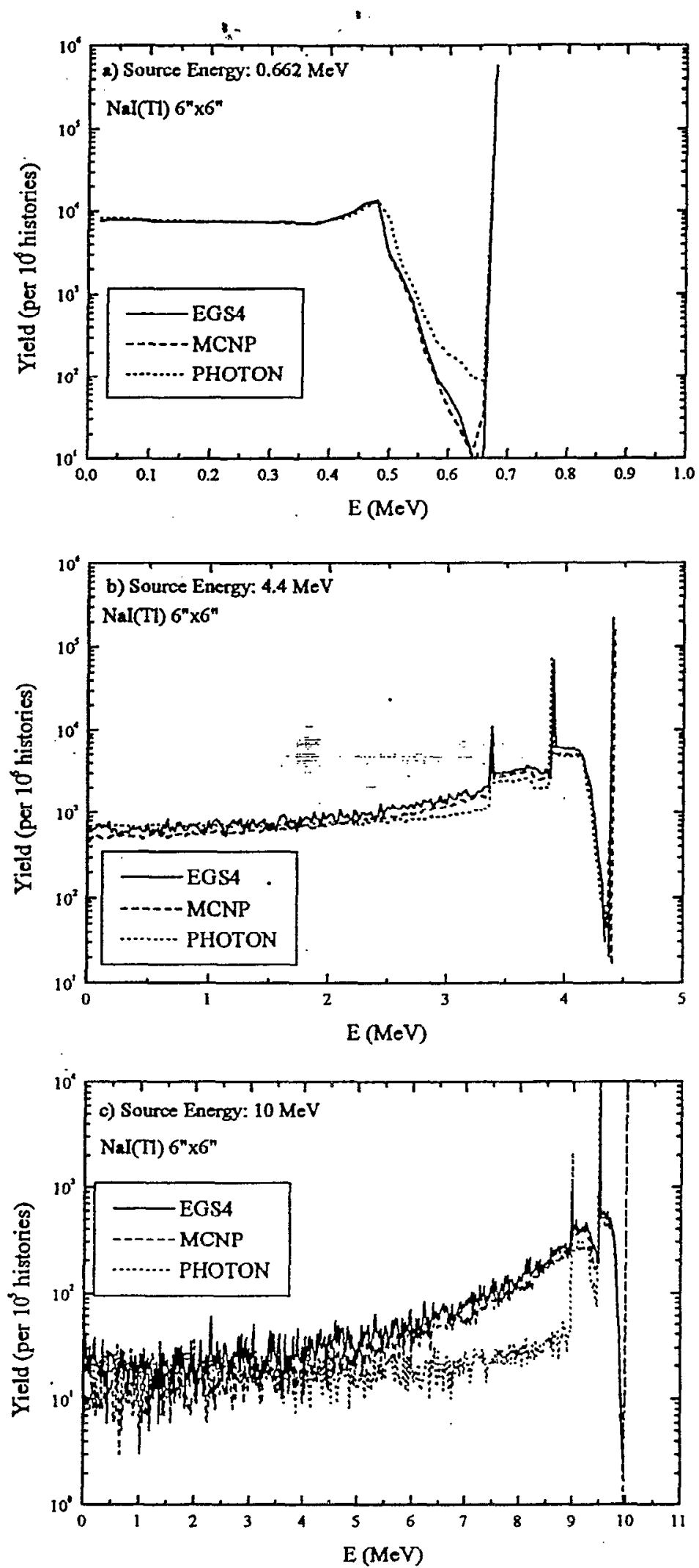

FIGURE 1. Comparison between the results of MCNP4B2, EGS4, and PHOTON Monte Carlo codes: (a) at a source energy of $0.662 \mathrm{MeV}$; (b) at $4.4 \mathrm{MeV}$; and (c) at $10 \mathrm{MeV}$. 


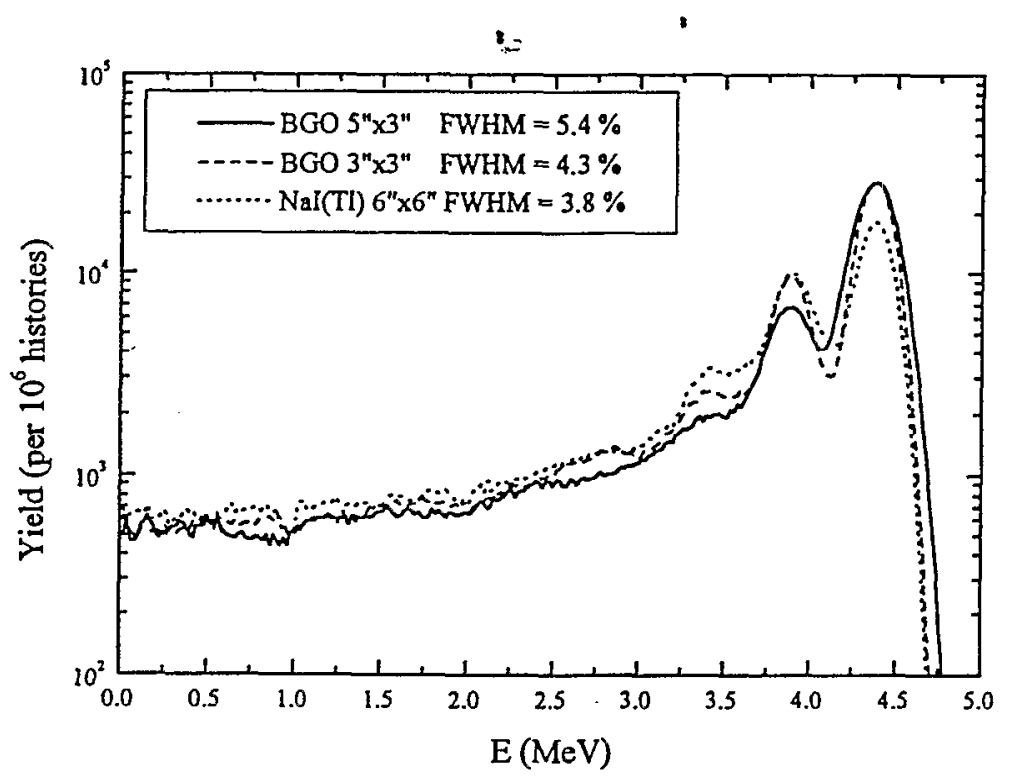

FIGURE 2. Simulated 4.4-MeV pulse-height distribution with the specific energyresolution function for BGO $5^{\prime \prime} \times 3^{\prime \prime}, \mathrm{BGO} 3^{\prime \prime} \times 3^{\prime \prime}$, and NaI(Tl) $6^{\prime \prime} \times 6^{\prime \prime}$ using the EGS4 Monte Carlo code.

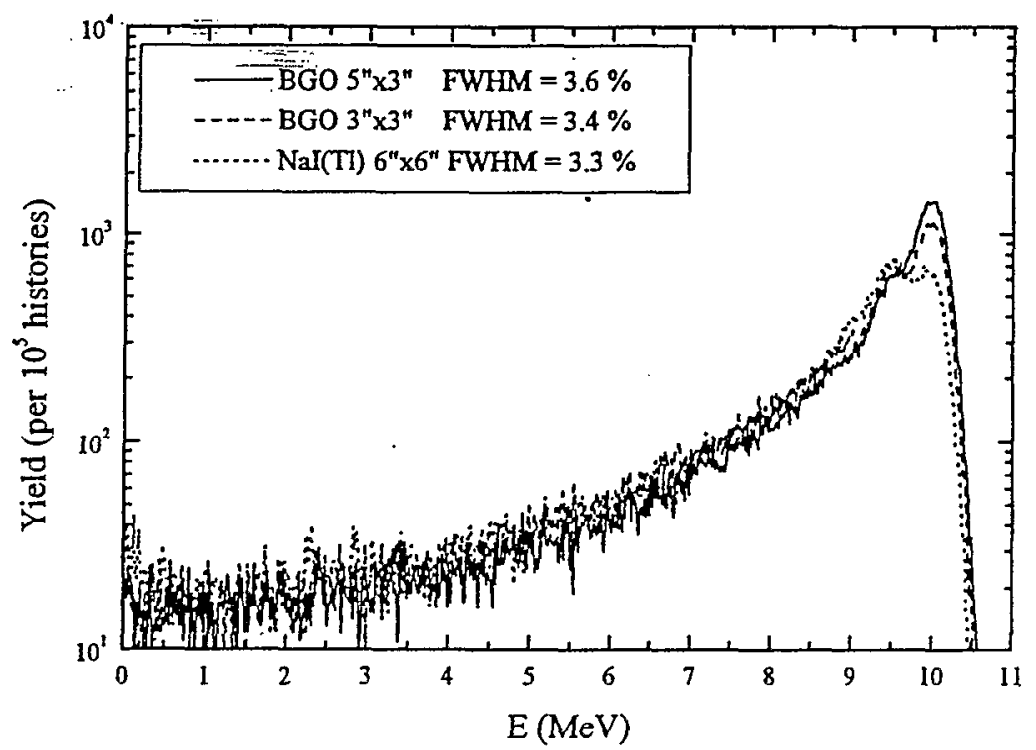

FIGURE 3. Simulated $10 \mathrm{MeV}$ pulse-height distribution with the specific energy resolution function for BGO $5^{\prime \prime} \times 3^{\prime \prime}$, BGO $3^{\prime \prime} \times 3^{\prime \prime}$, and NaI(Tl) $6^{\prime \prime} \times 6^{\prime \prime}$ using the EGS4 Monte Carlo code.

\section{DISCUSSION AND CONCLUSIONS}

At high energies, the higher energy resolution of the BGO detectors had little effect on the distribution of pulse height compared to that of the NaI detectors (FIGS. 2 and 3). However, the high efficiency of the BGO detectors led to a higher peak-tobackground ratio (FIG. 4). At higher energies the shape of the Compton region is al- 


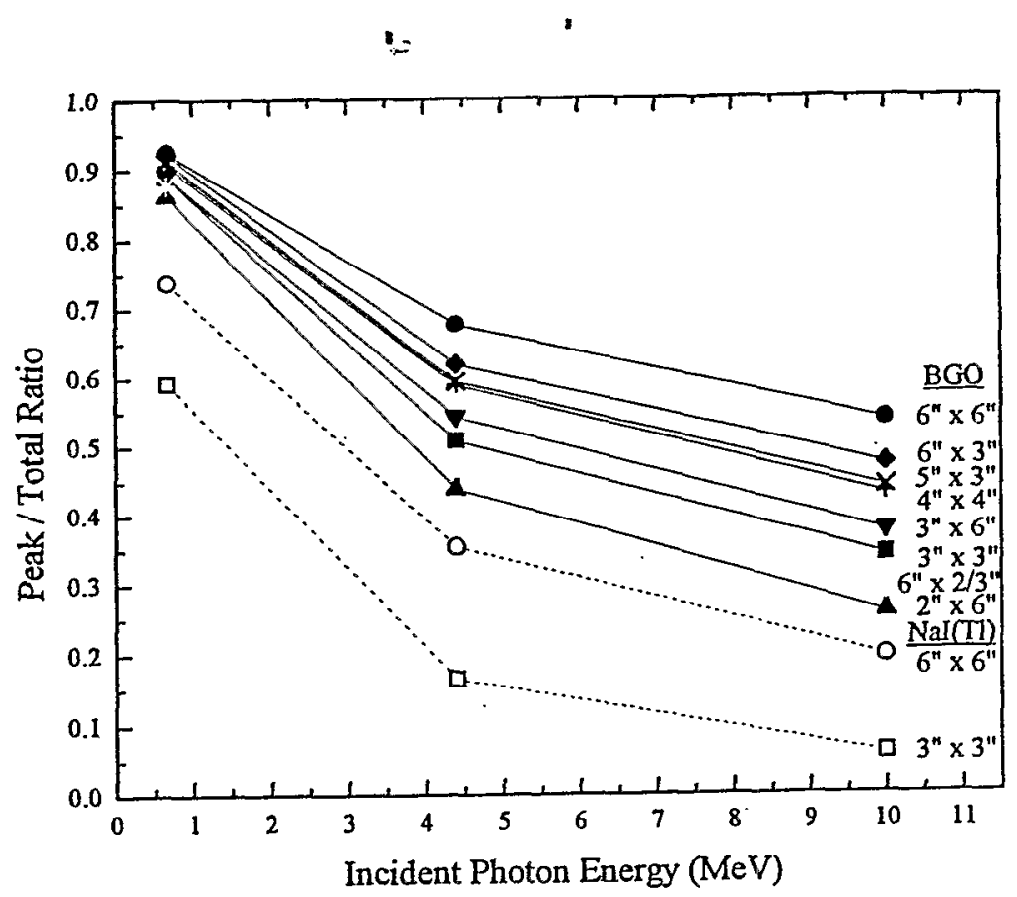

FIGURE 4. Calculated peak-to-total ratios versus incident photon energy for each detector by type-and by size.

TABLE 1. The yield per history of the single-escape (SE) and double-escape (DE) peaks and the photopeak-to-escape-peak ratios at two source energies

\begin{tabular}{|c|c|c|c|c|c|c|c|c|c|c|}
\hline \multirow[b]{2}{*}{ Detector } & \multicolumn{5}{|c|}{$\mathrm{E}=4.4 \mathrm{MeV}$} & \multicolumn{5}{|c|}{$E=10 \mathrm{MeV}$} \\
\hline & $\mathrm{P} / \mathrm{N}$ & SEN & $\mathrm{DE} / \mathrm{N}$ & $\mathrm{P} / \mathrm{SE}$ & $\overline{\mathrm{P} / \mathrm{DE}}$ & $\mathrm{P} / \mathrm{N}^{*}$ & $\mathrm{SE} / \mathrm{N}^{*}$ & $\mathrm{DE} / \mathrm{N}^{*}$ & P/SE & $\mathrm{P} / \mathrm{DE}$ \\
\hline \multicolumn{11}{|l|}{$\mathrm{NaI}(\mathrm{Tl})$} \\
\hline $3^{\prime \prime} \times 3^{\prime \prime}$ & 0.082 & 0.051 & 0.019 & 1.6 & 4.2 & 0.031 & 0.045 & 0.016 & 0.7 & 1.9 \\
\hline $6^{\prime \prime} \times 6^{\prime \prime}$ & 0.218 & 0.066 & 0.009 & 3.3 & 25.7 & 0.124 & 0.080 & 0.009 & 1.7 & 13.4 \\
\hline $\mathrm{BGO}$ & & & & & & & & & 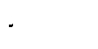 & \\
\hline $3^{\prime \prime} \times 3^{\prime \prime}$ & 0.354 & 0.074 & 0.006 & 4.8 & 62.3 & 0.240 & 0.078 & 0.007 & 3.1 & 35.1 \\
\hline $5^{\prime \prime} \times 3^{\prime \prime}$ & 0.427 & 0.064 & 0.004 & 6.7 & 112.4 & 0.323 & 0.070 & 0.004 & 4.6 & 72.6 \\
\hline
\end{tabular}

ABBREVIATIONS: $\mathrm{N}=10^{6}$, number of histories; $\mathrm{N}^{*}=10^{5}$, number of histories; $\mathrm{P}=$ photopeak counts; $S E=$ single escape peak net counts; $D E=$ double escape peak net counts.

most independent of the type and the size of the detector. For the BGO detector, the photopeak intrinsic efficiency versus detector volume is effectively represented by a single logistical equation of the form

$$
E_{f}=\frac{A 1-A 2}{1-V^{0.33}}+A 2,
$$

as shown in FIGURE 5. The main differences in the response function for the detectors of different size and type were in the escape peaks (see TABLE 1). In summary, the 


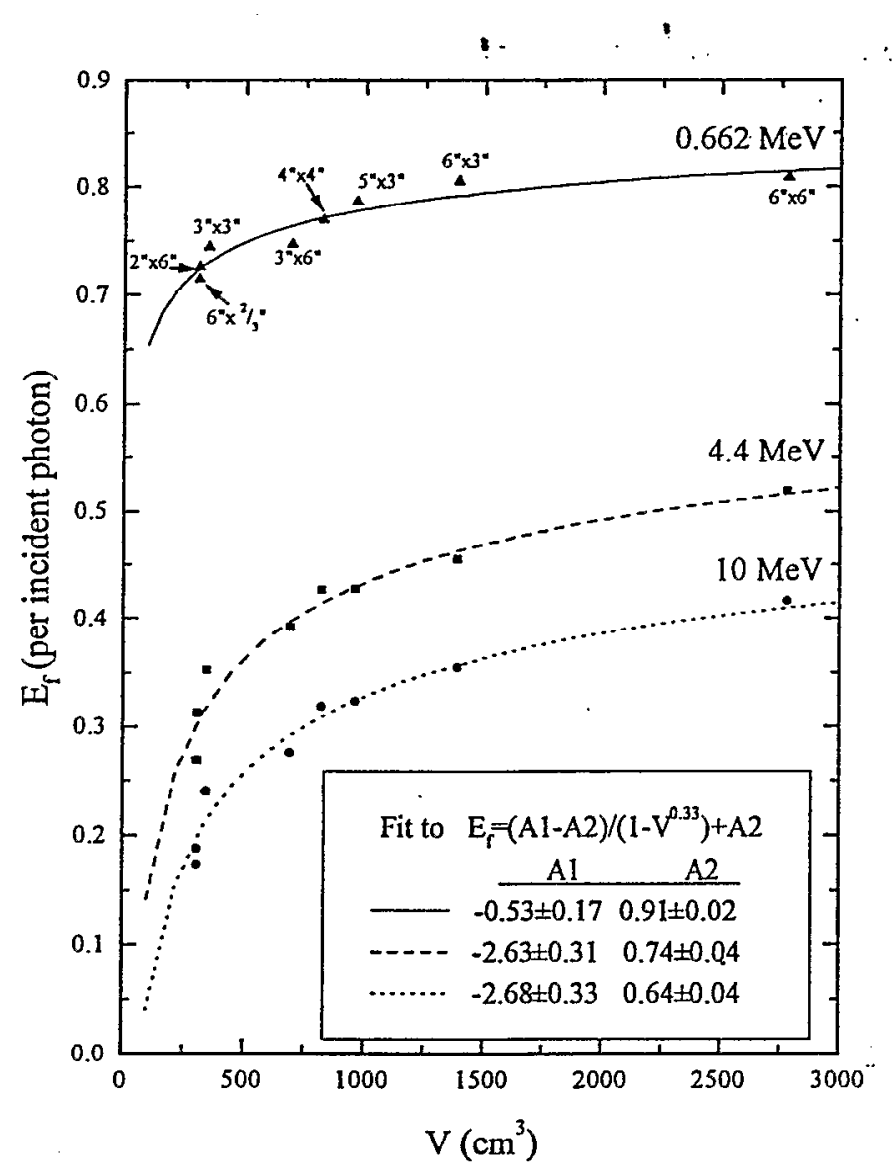

FIGURE 5. The BGO detector's intrinsic efficiency $\left(E_{f}\right)$ dependence on volume fitted to the logistical equation (the parameters are listed for three incident-photon energies).

BGO detectors, despite their worse energy resolution, appear to be superior to $\mathrm{NaI}(\mathrm{Tl})$ detectors for high-energy gamma detection.

\section{REFERENCES}

1. Nelson, W.R., H. Hirayama \& D. Rogers. 1985. The EGS4 Code. SLAC-265.

2. Briesmeister, J.F., Ed. 1997. The MCNP Transport Code, Version 4B. LA-12625-M.

3. Puzovic, J.M. \& I.V. ANICIN. 1998. User-friendly Monte Carlo program for the generation of gamma-ray spectral responses in complex source-detector arraignments. NIM. A 414: 279-282.

4. HEAT, R.L. 1964. Scintillation Spectrometry Gamma-Ray Spectrum Catalogue. Vol. 2, 2nd edit. U.S. Atomic Energy Commission IDO-16880-2. 